\title{
CFD FLOW ANALYSIS IN CENTRIFUGAL VORTEX PUMP
}

\section{INTRODUCTION}

One way of improving the characteristics of centrifugal pumps is by adding a vortex rotor to the centrifugal rotor by which energy of induced vortices at the vortex rim is added to fluid energy gained in centrifugal rotor, (Mihajlovič et al., 2001), (Isaakovič and Vasiljevič, 2003), (Karakulov et al., 2005), (Melzi, 2008) (Figure 1).

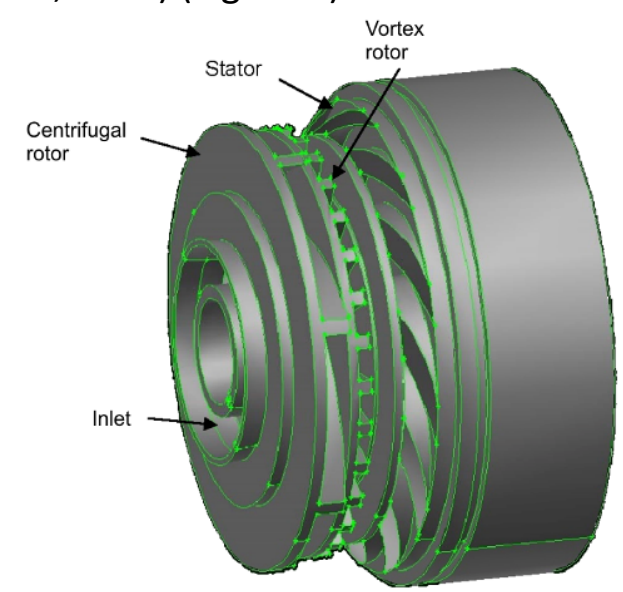

Figure 1: Centrifugal vortex pump

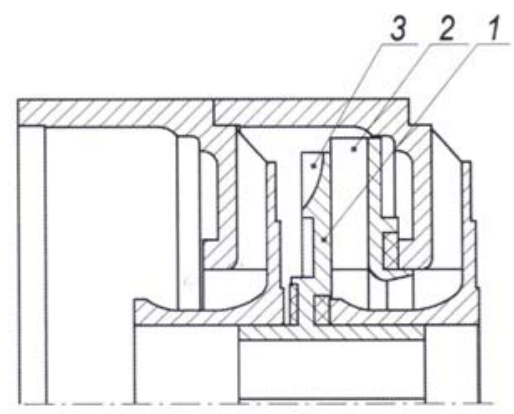

Figure 2: Cross section of the centrifugal vortex pump stage

Figure 2, shows a cross-section of the centrifugal vortex pump stage with annotated major components. On the periphery of the disc (1) of the centrifugal rotor (2) on the stator side, the vortex rim is installed (3).

The resulting additional kinetic energy of coherent structures induced at the vortex rim transforms to the head $\mathrm{H}_{4}$, which is added to the bulk head obtained in the centrifugal rotor of the centrifugal vortex pump $H_{\text {cen }}$ as shown in (1.1).

$$
H=H_{\text {cen }}+H_{4}
$$

Mere experiments do not provide all the necessary information about the structure of the energy conversions, (Bilus and Predin, 2009).

The objectives of this research were to clarify the mechanisms of energy conversion in a centrifugal vortex pump and to confirm the positive impact of a vortex rim on the pump characteristics. To do that, first aim was to generate a valuable numerical model which will provide a useful tool to study various cases. Numerical model should be robust and reliable 
to become a standard method in simulating flow and other physical phenomena occurring in centrifugal pumps and similar turbo machines.

\section{NUMERICAL MODEL OF CENTRIFUGAL VORTEX PUMP}

Numerical simulations of unsteady flow in centrifugal and centrifugal vortex pumps were carried out using commercial software which utilise control volume method. The simulation includes a whole centrifugal and centrifugal vortex pump stage including suction and discharge pipe (Figure 3). Between physical and numerical models, geometric, kinematic and dynamic conditions for similar work were satisfied.
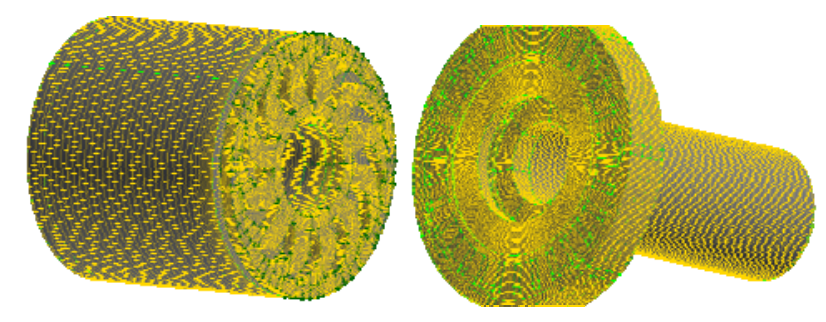

Figure 3: Discretization of centrifugal vortex pump

On the left side of Figure 3, the meshed stator with the outlet is shown, while the meshed rotor inlet pipe is shown on the right.

In the CFD model, the rotor rotates unrestrained, and is connected to the rest of the domain with a sliding mesh boundary condition, (Figure 4). The output tube is connected with the output from the stator with the interface boundary condition in order to mesh it with bigger control volumes than those by which stator is meshed, Figure 4, because there the flow is less demanding for simulation than the flow in the stator and rotor.
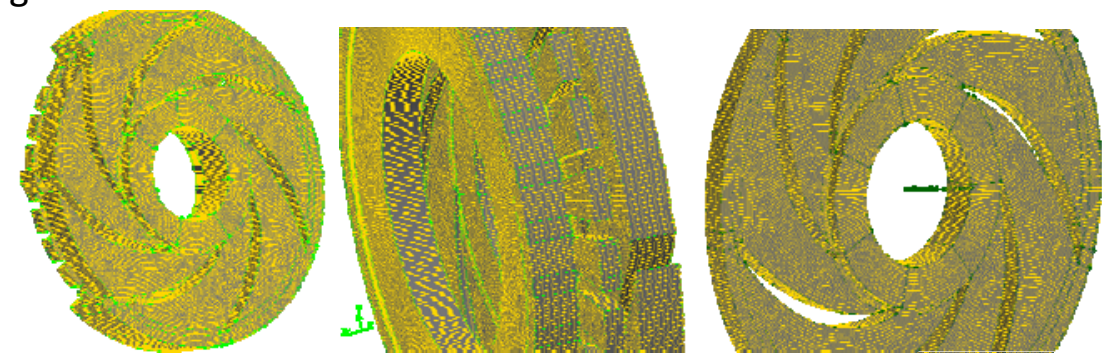

Figure 4: Meshed rotor

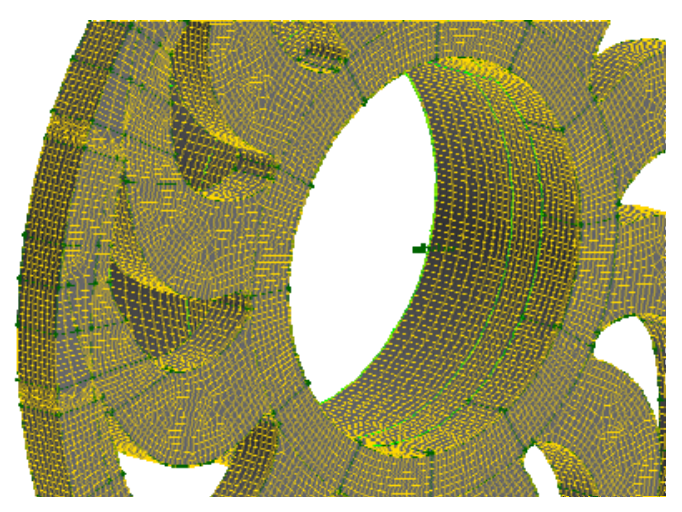


The entire continuum is meshed with three different meshes of control volumes. The first mesh consists of 962,159 cells and 108,640 nodes, with the greatest cell edge of $0.5 \mathrm{~mm}$; the second one consists of 1,864,399 cell and 2,007,183 nodes, with the greatest cell edge of 0.4 $\mathrm{mm}$; the third mesh consists of 3,340,658 cell and 3,573,140 nodes with the greatest cell edge of $0.3 \mathrm{~mm}$. Of the total number of control volumes, $85 \%$ of them were hexahedral control volumes, and $15 \%$ were mixed type cells, (Figure 5).

Given that the research was conducted at the rotor angular speed $n=2910 \mathrm{~min}^{-1}$, simulations were performed with a time step of $8 \times 10^{-5} \mathrm{~s}$. The time step was chosen after research for a suitable time step with respect to convergence, (Tucker, 1997). For the working fluid, water of standard properties at $25^{\circ} \mathrm{C}$ was used.

Turbulence was modelled using a hybrid DES SST model (detached eddy simulations) after it was been verified (sections 3 and 7). For the purpose of this work, a discretization scheme of second order was used.

Boundary conditions were set far enough from the pump stage so that their impact on the flow could be neglected. At the entrance to the domain, a pressure-inlet boundary condition was used, at the exit, an outlet-vent boundary condition was used, because it allows adjustment of the loss coefficient at the exit, (Kaupert and Staubli, 2003).

The boundary condition outlet vent is defined by pressure loss that is proportional to dynamic pressure:

$$
\Delta p=k_{L} \frac{1}{2} \rho v^{2}
$$

The outlet vent was also chosen because it best describes the physics of this research, in which the flow is regulated by the valve at the exit, from fully open to fully closed. Loss coefficient $k_{L}$ in the boundary condition outlet vent (5.1) is a number, and the entire simulation was conducted with $k_{L}=0$ (fully open valve), $2,5,10,20,60,300$ (fully closed valve), (Fluent Inc, 2008).

\section{SIMULATION OF DETACHED EDDIES (DES)}

Steady and unsteady models (RANS, URANS) are not suitable for numerical simulations of turbulent flows with significant separations (Figure 6). Furthermore, the computing of the problem in the application of the simulation of large eddies (LES) is highly demanding because of the necessity for high resolution mesh in thin boundary layers in the vicinity of the walls, to accurately solve fine turbulent structures occurring there, (Benim et al., 2010).

a)

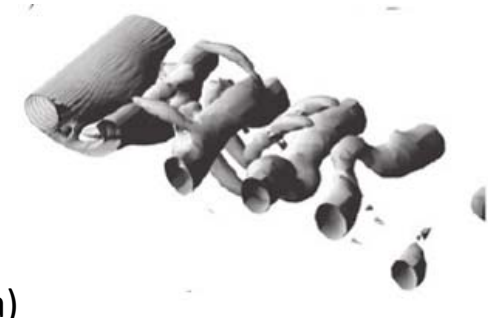

b)

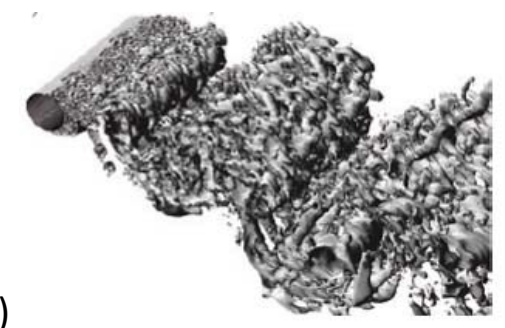

Figure 6 - Turbulent content depending on turbulence model in the flow around the circular cylinder. Display with isosurfaces of vorticity, $R e_{D}=50000$, experimentally established drag coefficient $C_{d}=1.15 \div 1: 25$ : a) $3 D$ unsteady RANS, SST $k-\omega, C_{d}=1.24$. The solution includes 
large separated vortices and clearly shows three-dimensional character: longitudinal rollers and transverse ribs; b) DES, fine mesh, the SST $k-\omega, C_{d}=1.28$., (Spalart, 2009)

This led to the development of hybrid URANS / LES methods in which the turbulence in the flow field that is in contact with wall is modelled with RANS turbulence models, while the flow in the core, away from the wall and in the areas of separations are solved with LES. Since of the all hybrid methods Detached-Eddy Simulation (DES) is the most developed and validated, it has been adopted in this work, (Travin et al., 1999). DES is an unsteady RANS simulation in which the turbulence model is modified so that, with the restriction of turbulent viscosity, the generation and development of large turbulent eddies enables.

DES was first proposed in 1997 (Shur et al., 1999) as a modification of a Spalart-Allmaras RANS turbulence model. The Spalart-Allmaras model as a linear scale uses the distance to the nearest wall $d_{\mathrm{w}}$ while the DES uses the value named "DES limiter":

$$
\tilde{d}_{\mathrm{w}}=\min \left(d_{\mathrm{w}}, C_{\mathrm{des}} \Delta_{\max }\right)
$$

where the constant $C_{\text {des }}=0.65$ and the characteristic size of the local computation point $\Delta_{\max }=\max (\Delta x, \Delta y, \Delta z)$ is defined as the biggest length of cell in either direction. Near the wall, the DES limiter value is reduced to the starting value of the $d_{\mathrm{w}}$. Away from the wall, the limiter is proportional to the size of the local computation cell. The limitation of length scales is indirectly limiting the growth of turbulent viscosity, thus enabling the development of small natural disturbances and the formation of turbulence. In other words, turbulent eddies in the attached boundary layer are modelled with the RANS turbulence model, while the larger detached eddies are simulated and solved. With this, the effect of reducing the impact of the RANS turbulence models in DES is achieved. This also reduces the uncertainty from imperfections of RANS modelling in the areas of flow. In contrast, RANS models are developed and give their best results when used in the boundary layers.

The DES based on the SST $k-\omega$ turbulence model that was used in this paper was proposed (Travin et al., 2002) in 2001. The DES SST $k$ - $\omega$ modification refers to a member of the $k$ equation that represents the dissipation of turbulent kinetic energy:

$$
\varepsilon=\beta * k \omega=\frac{k^{3 / 2}}{L_{t}},
$$

where the linear scale, the length of mixing is:

$$
L_{t}=\frac{\sqrt{k}}{\beta^{\star} \omega}
$$

DES modification of the expression (6.2) comes down to:

$$
\varepsilon=\beta^{\star} k \omega=\frac{k^{3 / 2}}{\min \left(L_{t}, C_{d e s} \Delta_{\max }\right)}
$$

where the constant $C_{\text {des }}=0.61$ for the SST model. The DES SST $k-\omega$ model is usually formulated as a multiplier dissipation member of the k-equation: 


$$
\varepsilon=\beta^{\star} k \omega F_{\text {des }}, F_{\text {des }}=\max \left(\frac{L_{t}}{C_{\text {des }} \Delta_{\max }}, 1\right)
$$

In both definitions $(6.1,6.5)$, DES suffers from the problem that was noticed in this work early and is the result of the premature activation of the DES limiter in the boundary layer. The "trigger" to activate the DES limiter is only the local mesh density. The fine grid in the area of the boundary layer can activate that DES limiter that will start to lower the effective viscosity, but at the same time, the grid can still lack sufficient finesse to enable the development of dissolved turbulent structures (LES content) in the attached boundary layer, (Fernández Oro et al., 2011). Because of this overly low turbulent viscosity, the Reynolds stress $\tau_{i j}{ }^{R}$ is also too low, which can cause the nonphysical boundary layer separation. The problem of premature activation of the DES limiter was solved by using the weight functions $F_{1}$ and $F_{2}$ of the SST $k-\omega$ model, (Menter and Kuntz, 2004). As the weight functions prevent the activation of $k-\varepsilon$ branch of the SST model, they also prevent activation of DES limiter within the attached boundary layer.

$$
F_{\text {des }}=\max \left(\frac{L_{t}}{C_{\text {des }} \Delta_{\max }}\left(1-F_{S S T}\right), 1\right) \text {, with } F_{S S T}=0, F_{1}, F_{2}
$$

$F_{S S T}=0$ brings out original formulation without the protection of the boundary layer (6.5). The function $F_{2}$ provides stronger protection of boundary layer and is therefore a preferred choice. The protection of the boundary layer is not absolute, but reduces the risk of premature activation of LES branch by an order of magnitude. It was shown, that the local maximum dimension of computational cell on the wall surface not less than $10 \%$ of the thickness of the boundary layer at the same position, gives the best results.

\section{VALIDATION OF THE USED NUMERICAL MODEL}

With the process of validation to what degree solved mathematical model approximates reality, can be determined.

Figure 7 shows the process of validation of the numerical solutions for $Q-H$ characteristic of the centrifugal vortex pump stage. Validation is performed by comparing numerical solutions with the experimental solution.

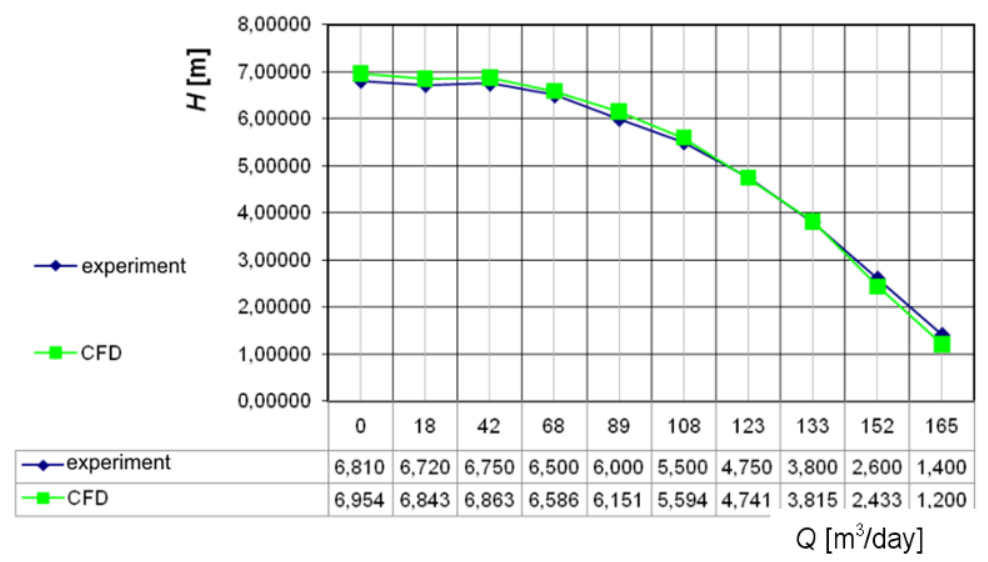

Figure 7: Q-H characteristics of centrifugal vortex pump stage, obtained by CFD and experiment 
Figure 7 shows very good agreement of CFD results with experimental results. It is evident that the CFD in the zone from the $165 \mathrm{~m}^{3} /$ day $(1.91 \mathrm{l} / \mathrm{s})$ to $123 \mathrm{~m}^{3} /$ day $(1.42 \mathrm{l} / \mathrm{s})$ falls short in comparison to the experiment, which is attributed to the lack of used computational domain.

From the flow rate of $108 \mathrm{~m}^{3} /$ day $(1.25 \mathrm{l} / \mathrm{s})$ to $0 \mathrm{~m}^{3} /$ day, the CFD solution exceeded the experimental solution, which is a computational simulation error that has been reported, (Launder and Spalding, 1972). According to (Feng et al., 2009), CFD falls short in predicting the intensity of turbulence, but it generates higher velocity fields in the radial space between the rotor and stator. Since this space is of crucial importance for the centrifugal vortex pump, this is a valid explanation for the larger $H$ in this range of the flow. Specifically, generated flow with these higher velocity fields encounters the vortex rim, which then generates more energy, so fluid with increased energy enters the diffuser, which then generates higher $\mathrm{H}_{4}$ according to Equation (1.1), resulting in higher $\mathrm{H}$.

However, since the maximum deviation of head is $0.15 \mathrm{~m}$ with a flow rate of $89 \mathrm{~m}^{3} /$ day $(1.03$ $\mathrm{l} / \mathrm{s}$ ), which was $2.5 \%$ of the head at that flow, and since the average deviation of head throughout the whole working scope was $0.09 \mathrm{~m}$, a CFD solution is considered that has welldescribed covered physics of current flow.

\section{VALIDATION OF THE USED MODEL OF TURBULENCE}

Figure 8 shows the $Q-H$ characteristics of centrifugal vortex pump obtained by CFD simulations on the same mesh with the same time step and discretization schemes, but with different models of turbulence. The curve labelled "CFD centrifugal vortex pump" is the solution obtained by the DES turbulence model, for which "error estimate" has been conducted (sections 4 and 6 ), so it is to be considered the correct numerical solution. From Figure 8 it can be seen that the solutions obtained with the other four models of turbulence $(k-\varepsilon, k-\varepsilon$ RNG, $k-\omega$ SST and RSM) really fall short in predicting the head of centrifugal vortex pump; in fact, they resemble the solutions for the centrifugal pump (Figure 11). This means that other models of turbulence are not able to cover the effect of the vortex rim. From this, we can conclude that the mechanism of energy conversion from vortex rim largely depends upon the energy of coherent vortex structures generated by its blades. These coherent structures are severed from the vortex rim and they give their energy to the main fluid flow coming from the centrifugal impeller by the mechanism of increasing kinetic energy. 


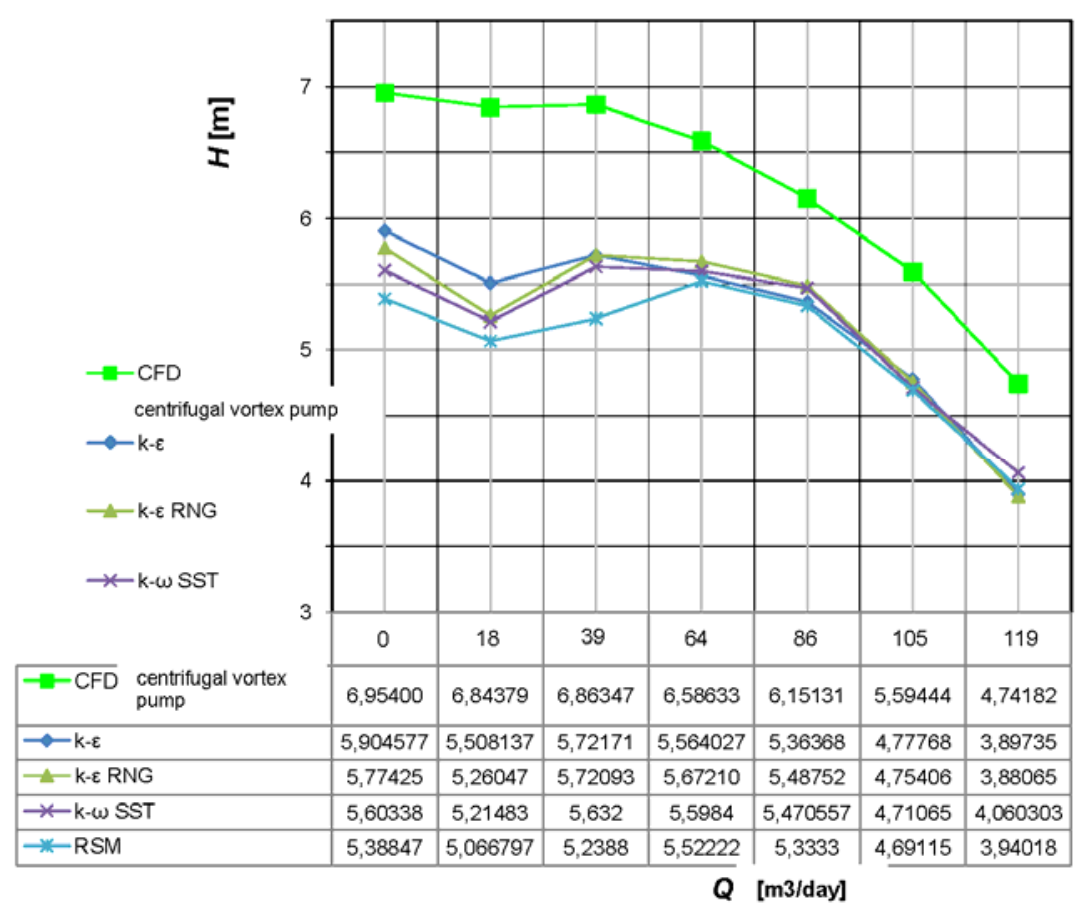

Figure 8: $Q-H$ characteristics of centrifugal vortex pump stage, obtained by different turbulence models

Vortex chamber, area between vortex rim and stator (Figure 2), limits size of eddies. Since most of the turbulent energy is contained in the largest scale eddies and the eddy size is limited by the distance from the wall, the near-wall energy containing eddies will be small while those centred in the core will be large. The transfer of energy from the small to large scales is an example of an inverse energy cascade as opposed to the classical energy cascade. Figure 6 shows that DES turbulence model solve vortices in separation areas, while other turbulence methods models those vortices thus average their energy. So DES is able to pick up part of the energy of this inverse energy cascade which is lost in other turbulence models. This is more pronounced at the low flow rates cause in this working regime vortex rim creates lots of coherent structures. At the high flow rates vortex rim do not generate coherent structures but DES still shows superiority over other turbulent models cause it thoroughly captures energy contained in eddies of a main flow coming from centrifugal rotor.

\section{VERIFICATION OF THE USED NUMERICAL MODEL}

The method to verify the numerical models used, determines that they are solved mathematically correctly. To treat any information obtained by experiment and numerical simulation as a result, it is necessary to know how certain we can be in it or (in other words) what its uncertainty is. The uncertainty is the estimated amount by which the calculated results may differ from exact solution.

$$
\text { Exact solution }=\text { Calculated result } \pm \text { estimated uncertainty }
$$

The results uncertainties are comprised of: 1) Uncertainty of input data, 2) Uncertainty of the used mathematical models, and 3) The numerical uncertainty. Numerical uncertainty is the only element that cannot be eliminated. Its sources are in errors due to insufficient 
spatial discretization resolution, in the temporal discretization errors due to the inadequate time step, in the incomplete convergence of iterative solver, and in the errors in computational software.

The method of determining the spatial discretization errors used in this study is comprised of calculating three solutions on three different mesh sizes, (Roache, 1997), (NEA - Committee on the safety of nuclear installations, 2007). The first step was to determine referent size of control volume $\Delta$ by:

$$
\Delta=\left[\frac{1}{N} \sum_{i=1}^{N}\left(\Delta V_{i}\right)\right]^{1 / 3}
$$

where: $\Delta V_{i}$ is the volume of $\mathrm{i}^{\text {th }}$ cell, $N$ is the total number of cells by which the domain is meshed. Then suppose that $\Delta_{1}<\Delta_{2}<\Delta_{3}$ and $r_{21}=\Delta_{2} / \Delta_{1}, r_{32}=\Delta_{3} / \Delta_{2}$ The "apparent" order of accuracy $p$ can be calculated using the following equations:

$$
\begin{gathered}
p=\frac{1}{\ln \left(r_{21}\right)}|\ln | \varepsilon_{32} / \varepsilon_{21}|+q(p)| \\
q(p)=\ln \left(\frac{r_{21}^{p}-s}{r_{32}^{p}-s}\right) \\
s=1 \cdot \operatorname{sgn}\left(\varepsilon_{32} / \varepsilon_{21}\right)
\end{gathered}
$$

where $\varepsilon_{32}=\Phi_{3}-\Phi_{2}$ and $\varepsilon_{21}=\Phi_{2}-\Phi_{1}$. If $r=$ const. then $q(p)=0$. The absolute value in (8.3) is needed to ensure that extrapolation asymptotically tends towards $\Delta=0$. Negative values of $\varepsilon_{32} / \varepsilon_{21}<0$ are indicators of oscillatory convergence. If possible, the percentage of occurrence of oscillatory convergence also should coincide with the "apparent" order of accuracy. This coincidence may be taken as a good indicator that the arrangements of control volumes are in the asymptotic region, but in case that coincidence is absent, it should not necessarily be taken as a sign of poor convergence. If $\varepsilon_{32}$ or $\varepsilon_{21}$ are "very close" to zero, the procedure does not work. This may be an indicator of overall oscillatory convergence, or in rare cases it may mean that the "correct" solution has been achieved. In such cases, if possible, calculations on the additionally refined mesh should be carried out; if not, the results can be accepted.

After that, the extrapolated value of the result, for which uncertainty is calculated, can be obtained with:

$$
\Phi_{\text {ext }}^{21}=\left(r_{21}^{p} \Phi_{1}-\Phi_{2}\right) /\left(r_{21}^{p}-1\right)
$$

Calculate the estimated error from:

$$
e r_{a}^{21}=\left|\frac{\Phi_{1}-\Phi_{2}}{\Phi_{1}}\right|
$$

Calculate the relative extrapolated error from:

$$
e r_{e x t}^{21}=\left|\frac{\Phi_{e x t}^{12}-\Phi_{1}}{\Phi_{e x t}^{12}}\right|
$$

Calculate the grid convergence index on the finest mesh, $\mathrm{GCl}_{\text {fini }}{ }^{21}$ from:

$$
\mathrm{GCl}_{\text {fini }}^{21}=\frac{1.25 e r_{a}^{21}}{r_{21}^{p}-1}
$$


The factor of 1.25 in the equation is actually a safety factor, which was to be more conservatively replaced by 3 in this work. If the value of this factor is 1 , it is analogous to a certainty of $50 \%$ that the result of numerical simulations fall into this area. Therefore, the safety factor of 3 corresponds to $99.73 \%$ (normal distribution) that the exact CFD solution lies in the range $\Phi_{\text {ext }} \pm \mathrm{GCl}$.

The previously described procedure of error estimate and given equations (8.4) to (8.11) can be used for time steps error estimate if the referent spatial dimension of the meshes $\Delta$ is replaced by time steps.

Figure 9 shows a CFD solution for the delivery head of centrifugal vortex pump generated from the results on three different meshes by the process of error estimate. The local order of accuracy $p$ is has varied from 1.53 to 2.20 , with a global average of $p=1.92$, which is a good indicator of computer simulations carried out. Oscillation convergence occurs in $40 \%$. The global order of accuracy is used to estimate the numerical uncertainty, or $\mathrm{GCl}$ (global convergence index). The maximum uncertainty of discretization is $2.88 \%$, which corresponds to the amount of head of $\pm 0.20 \mathrm{~m}$. Uncertainty is shown in the figure as a range of errors of each numerical result.

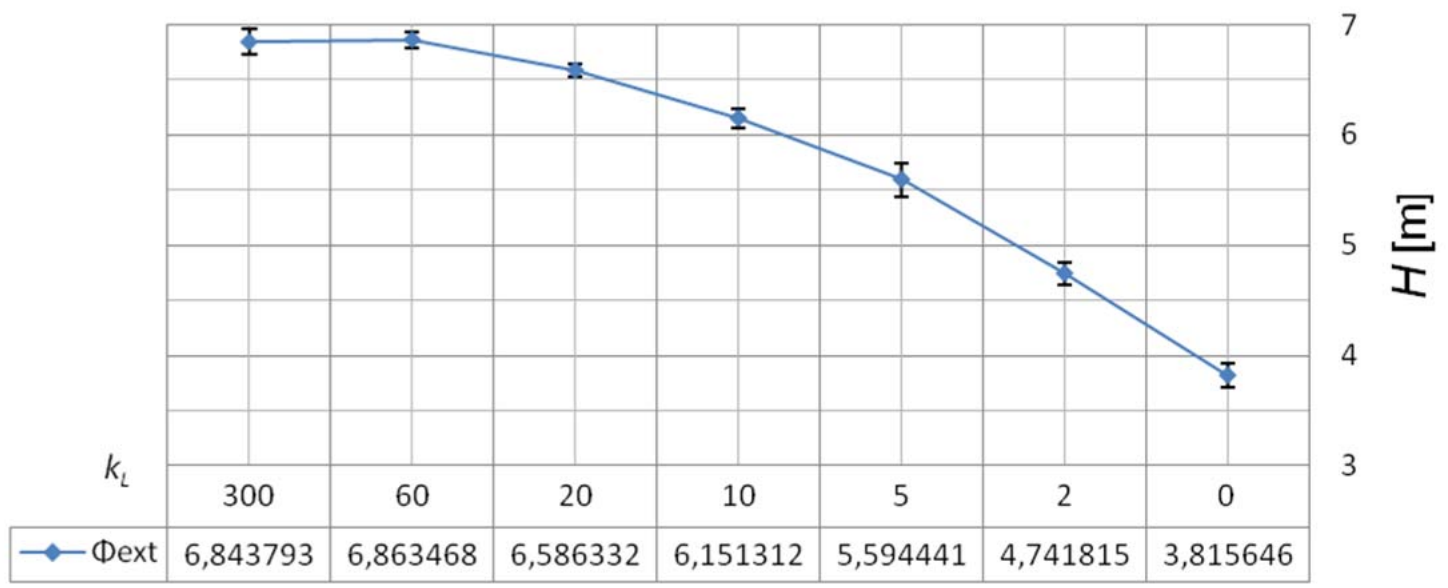

Figure 9: Extrapolated Q-H characteristics of centrifugal vortex pump stage with uncertainty bars - spatial error estimate

Figure 10 shows a comparison of CFD solutions for the pump delivery head obtained on the same mesh at three different time steps. The local order of accuracy $p$ is calculated by the equation (8.5) and it varies from 1.45 to 5.20, with a global average of the middle of $p=3.03$. The global order of accuracy is used to estimate the numerical uncertainty, or $\mathrm{GCl}$ index according to previous described procedure. Uncertainty is plotted in a range of errors, as shown in the figure. The maximum uncertainty of discretization is $1.57 \%$, which corresponds to the amount of supply of $\pm 0.11 \mathrm{~m}$. 


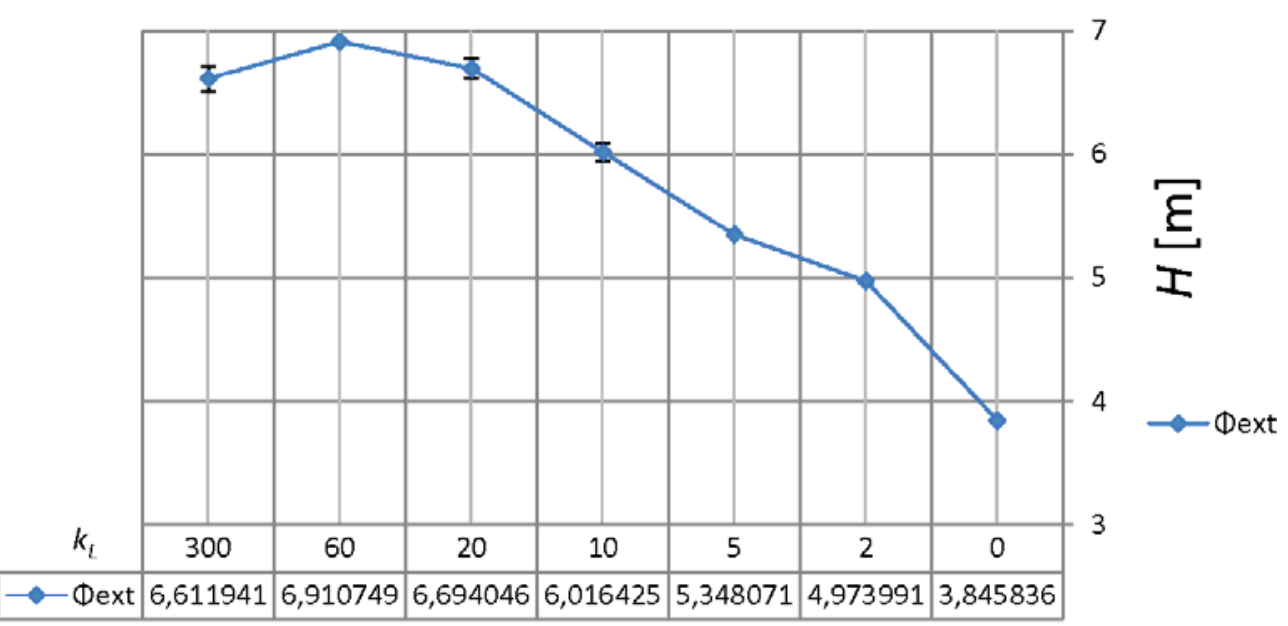

Figure 10: Extrapolated $Q-H$ characteristics of centrifugal vortex pump stage with uncertainty bars - time steps error estimate

The previously conducted error estimate has proven that the domain has been meshed with a sufficient number of cells with good refinements in demanding areas of flow. Any further refinement will not change results significantly. Furthermore, it has been shown that time step has been chosen correctly.

\section{RESULTS OF THE NUMERICAL AND EXPERIMENTAL RESEARCH OF CENTRIFUGAL VORTEX PUMP}

\subsection{COMPARISON OF Q-H CHARACTERISTIC OF CENTRIFUGAL AND CENTRIFUGAL VORTEX PUMP}

Figure 11 shows the $Q-H$ characteristics of a centrifugal pump stage and the $Q-H$ characteristics of a hybrid centrifugal vortex pump stage. It is evident that the characteristics of the centrifugal vortex pump smoothly follow the characteristics of centrifugal pump from the maximum flow to the flow of $105 \mathrm{~m}^{3} /$ day $(1.22 \mathrm{l} / \mathrm{s})$. After the flow of $105 \mathrm{~m}^{3} /$ day $(1.22$ $1 / s)$, the characteristics of the centrifugal vortex pump continue to rise steeply as a characteristic of vortex pumps in (Dochterman, 1974), in contrast to the characteristics of centrifugal pump, which grows slower than the characteristics of the centrifugal vortex pump to its maximum at flow rate of $65 \mathrm{~m}^{3} /$ day $(0.75 \mathrm{l} / \mathrm{s})$, and then begins to decline steeply. This form of performance curve represents the instability of the pump, because the pump can generate two different flow rates at a given head, left and right from the maximum $Q-H$ characteristic curve, (Lobanoff and Ross, 1992). 


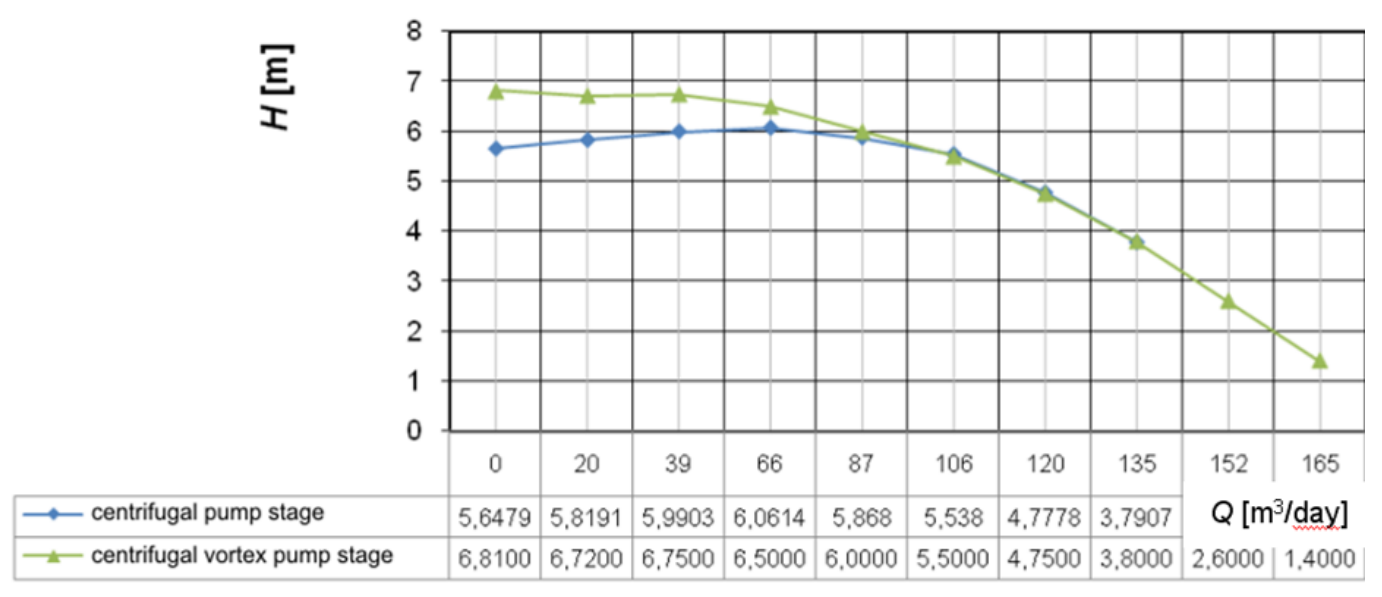

Figure 11: Q-H characteristics of centrifugal vortex and centrifugal pump

Adding a vortex rotor to the centrifugal pump stage provided a steeply sloping $Q-H$ characteristic pump, which ensures the stability of its operation. This confirmed one of the positive roles of the vortex rim in the centrifugal vortex pump stage.

\subsection{QUANTIFICATION OF THE PORTION OF HEAD GENERATED BY VORTEX ROTOR IN THE HEAD OF CENTRIFUGAL VORTEX PUMP}

The contribution of eddy processes on the amount of the head and the corresponding increase in pressure depends on the flow rate, and the given flow rate depends on the magnitude of the axial component of absolute velocity of the fluid from the discharge of centrifugal rotor to the entrance in the diffuser channels. The lower the flow rate and the smaller the axial velocity component are, the greater the number of the vortex rim passages participating in the change of momentum between the fluid contained in these passages at that instant of time and the main stream of the fluid from the centrifugal rotor to the diffuser, (Mihalić et al., 2011).

Figure 12 clearly shows that the vortex rim in the centrifugal vortex pump stage begins to increase the amount of the head from the flow rate of $105 \mathrm{~m}^{3} /$ day $(1.22 \mathrm{l} / \mathrm{s})$ to the zero flow rate, which is very convenient, because if there is a higher demand on the pump, so as to overcome the greater resistance, the vortex rim is increasingly helping the pump.

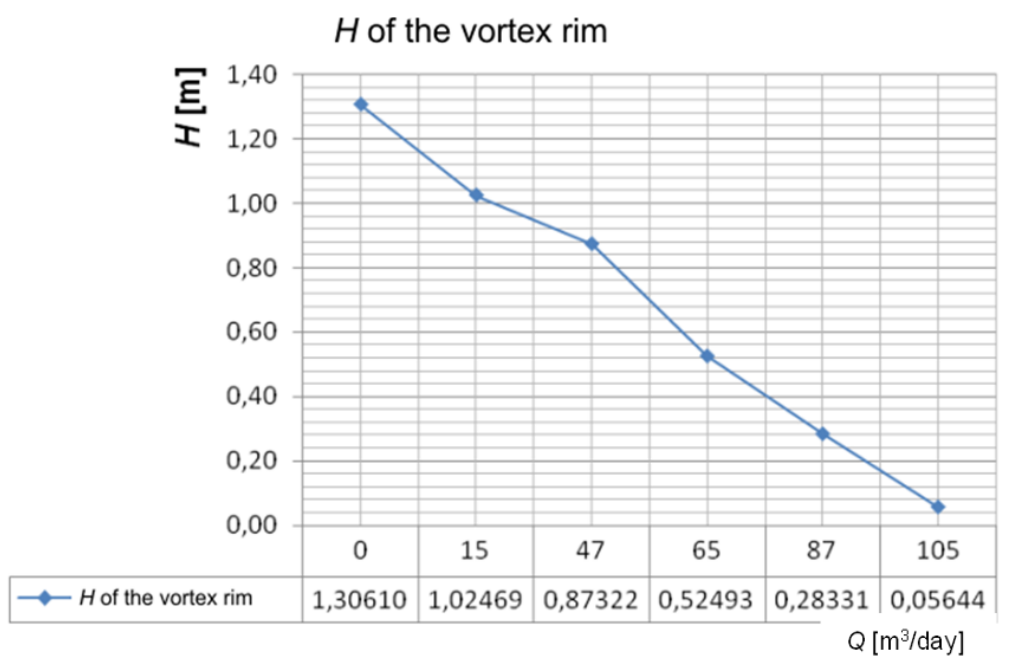

Figure 12: Contribution of the vortex rim to the increase of head $\mathrm{H}$ 
The swirl effect achieved by vortex rim increased the magnitude of the head of the plain centrifugal pump stage for a maximum of $23.13 \%$ (average increase of $11.64 \%$ ).

\subsection{COMPARISON IN THE EFFICIENCY OF CENTRIFUGAL VORTEX AND CENTRIFUGAL PUMP STAGE}

Figure 13 shows a comparison of efficiency curves of the centrifugal vortex and centrifugal pump stage researched in this work. It can be assumed that both curves shows a greater efficiency than would be present with the utilization of actual pumps. The reason for this is that the numerical models do not take into account friction losses due to wall roughness, and losses due to fluid flow through clearances (Gülich, 2008). However, we assumed that the numerical models of real pump geometry will calculate efficiency curves with the realistic trends and mutual correlations.

Efficiency of the centrifugal vortex and centrifugal pump stage
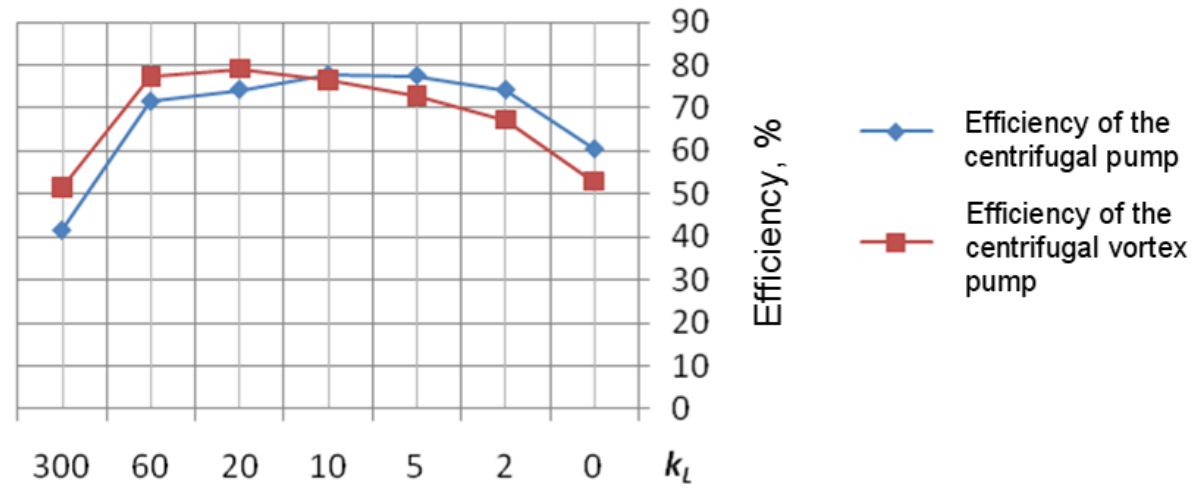

Figure 13: The efficiency curve of centrifugal vortex and centrifugal pump

A comparison of the efficiency curves (Figure 22) shows that at high flow rates $\left(k_{L}=0\right.$ to 10 ) centrifugal pump obtained greater efficiency than centrifugal vortex pump. The vortex rotor at high flow rates does not get enough fluid from the main flow (coming from the centrifugal rotor, Figure 15) so it does not increase the head (Figure 11), while at the same time it contributes significantly to the increase of entropy. In that working range, the vortex rotor only creates turbulence, and its kinetic energy is converted into a losses. Thus, in this working range, the vortex rotor is "parasitic part" in the centrifugal vortex pump.

In contrast, at low flow rates $\left(k_{L}=10\right.$ to 300$)$ the efficiency of the centrifugal vortex pump is greater than that of the centrifugal pump. In this working range, the vortex rotor grip sufficient amount of fluid from the main stream and contributes to increasing the level of head (Figure 11). Its kinetic energy is not transferred entirely to the creation of fluid turbulence, but a part of its kinetic energy is used to create a coherent flow structures and to accelerate the flow of secondary stream (Section 7.4).

It is also evident from Figure 13 that the best efficiency point of the centrifugal vortex pump is shifted to the left (to the smaller flow rates, and higher head) in comparison to the centrifugal pump. 
Figure 14 shows the flow through a centrifugal vortex pump; the flow structures can be observed. It can be seen that the flow through inlet pipe is even, steady, nearly laminar with pre-vortex at the entrance to the rotor. It is evident that after vortex rotor, flow becomes substantially vertiginous with many coherent structures. In the stator flow it becomes somewhat calm, but still there are vortices, which are now fewer and with larger diameters. From the figure, it can also be observed that some channels are choked with vortex while in others there are no vortices whatsoever. Furthermore, in other channels vortex is moving out from them. This phenomenon of the vortex appearing to be one and the same vortex travelling centrifugally from one rotor channel to another represents a traveling vortex instability and loss of energy, and is called "flow cutoff" or "rotating stall" (Matijašević et al., 2006).

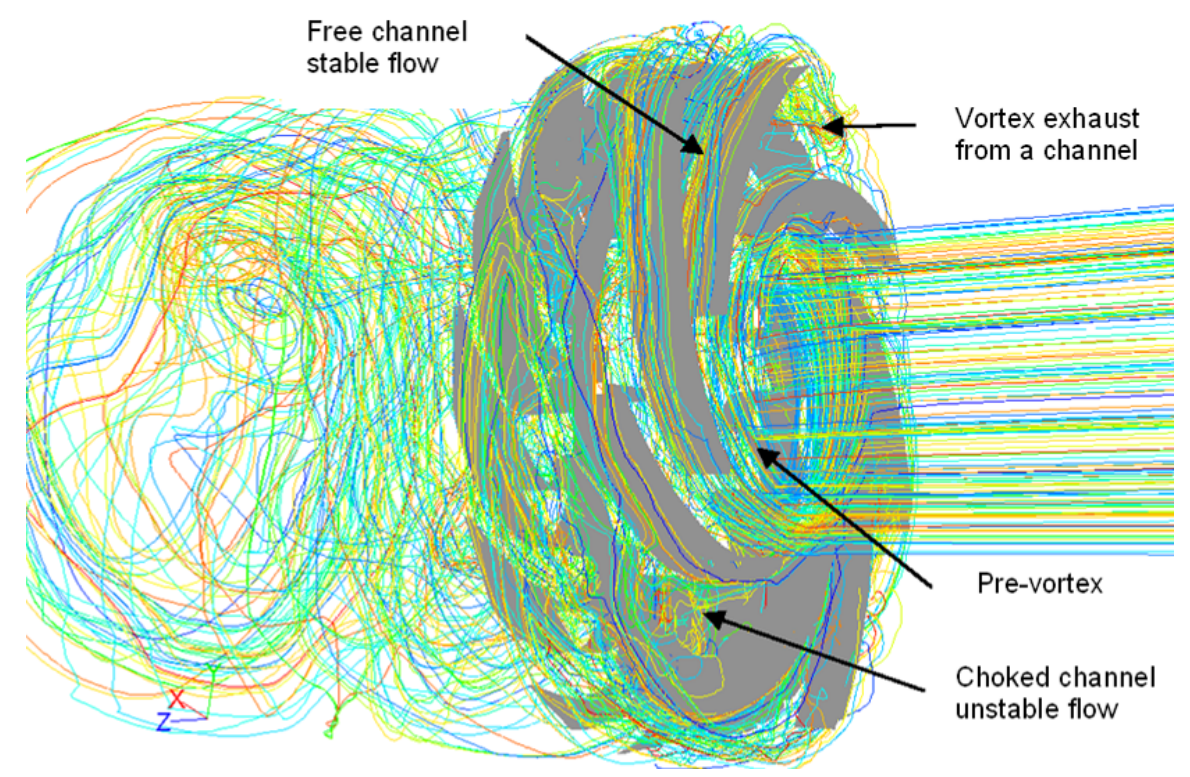

Figure 14: Path lines in the centrifugal vortex pump $k_{L}=60$

\subsection{ANALYSIS OF THE SECONDARY FLUID FLOW}

In Figure 15, the velocity vectors in the axial plane of the researched centrifugal vortex pump are shown. It can be seen the formation of the main flow of fluid coming from the centrifugal rotor and a secondary flow that comes from vortex rotor. Part of the main flow is taken by the vortex rotor, which then creates a secondary flow. 


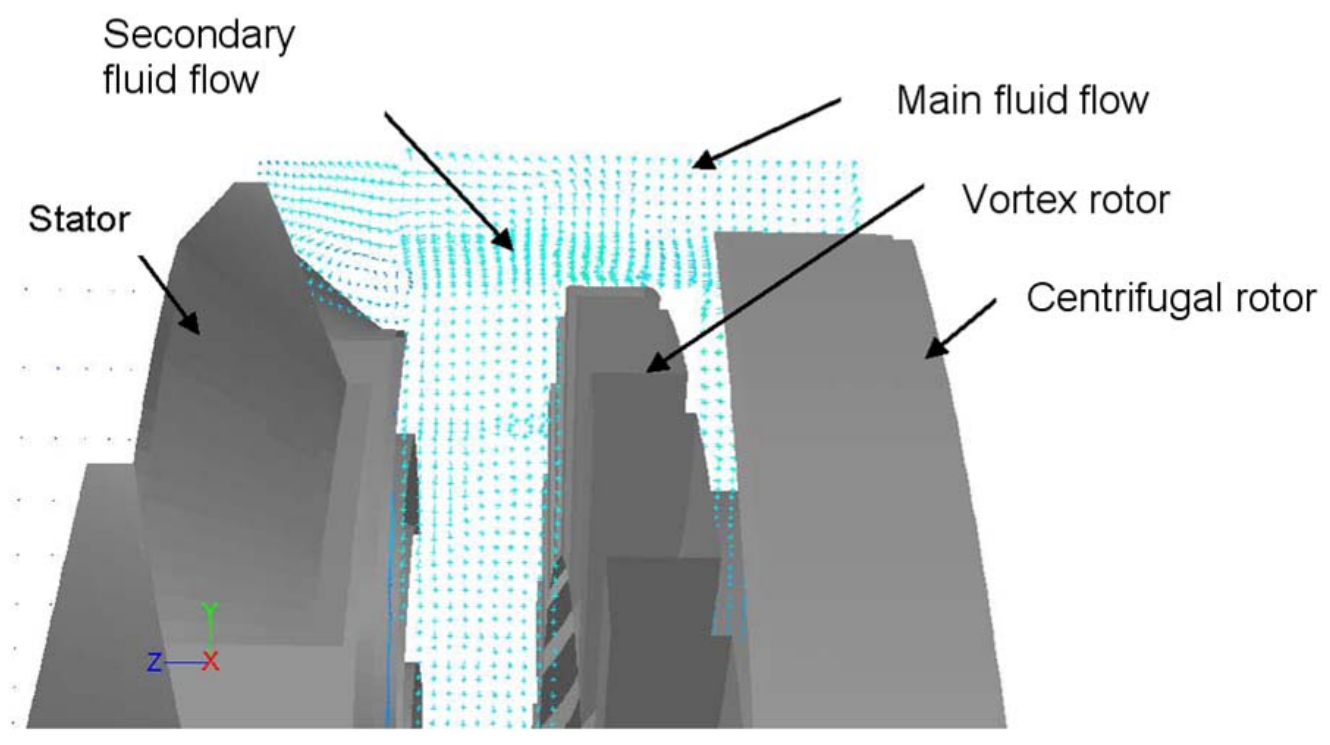

Figure 15: Main and secondary flow of fluid, $k_{L}=60$

Looking at Figure 15, it can be concluded that part of the main flow that comes out from centrifugal rotor blades approaching the stator loses its kinetic energy, and fails to enter the stator, but gets pushed down (towards lower pressure) from the inlet in the stator to the axis of rotation. At that time, the secondary flow is created from this part of main fluid flow. When this secondary fluid flow arrives to the root of the vortex rotor blades, they take it and push it to the periphery (towards larger radius) by the centrifugal force (Figure 16). Given that the movement toward larger radius increases the angular velocity of the secondary flow, its kinetic energy increases. With the increased kinetic energy, the secondary flow collides with the main flow, transferring additional kinetic energy to it. The main flow with that increased energy enters into the stator, ultimately generating an increased had of th centrifugal vortex pump relative to the centrifugal pump without that mec secondary fluid flow energy transfer.

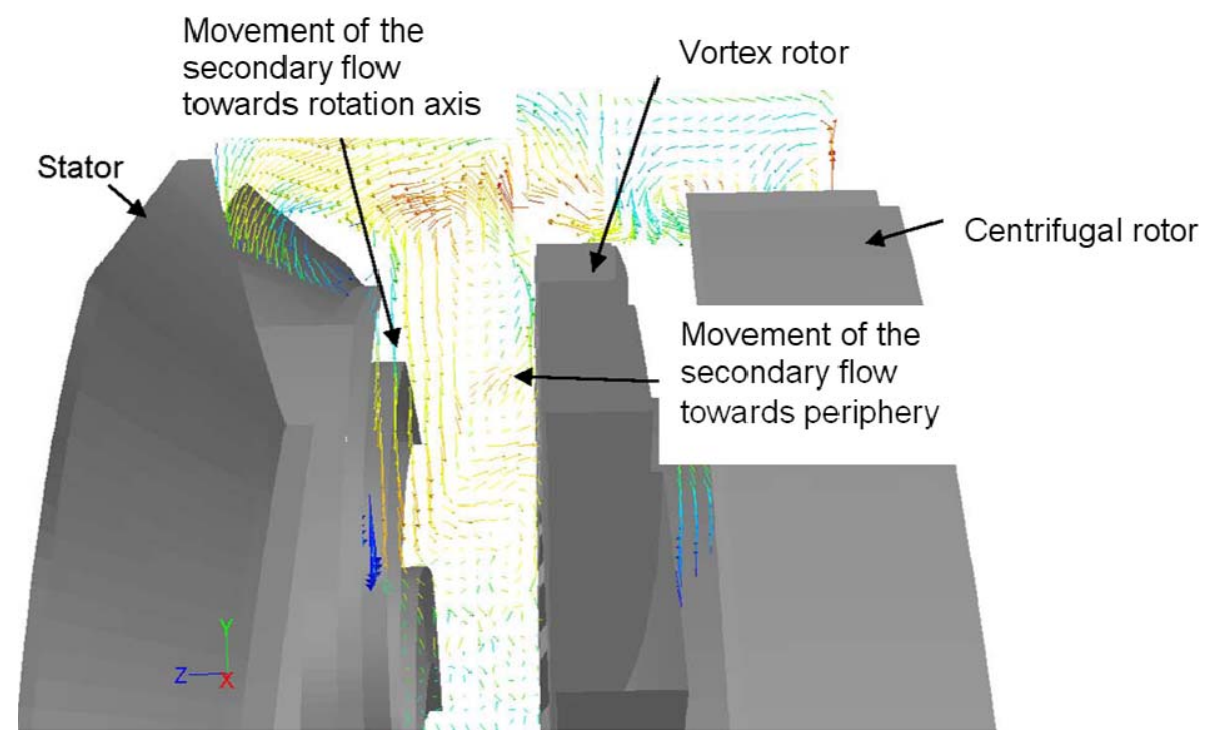

Figure 16: Movement of the secondary fluid flow, $k_{L}=60$ 


\section{CONCLUSION}

Numerical control volume method with unsteady solver and DES turbulence model was proven to be valuable tool for flow analysis in centrifugal pump. Having in mind, that DES turbulence model consumes much less computational time than LES turbulence model, this is very useful fact that resulted from this research.

It was proven that the kinetic energy of coherent vortex structures created by the vortex rim is added to the fluid flowing from the passages of the centrifugal rotor and thus increases the total energy of the fluid before entering the stator. Furthermore, this extra energy is added to the main fluid stream by the longitudinal vortices generated by the edges of the vortex rim due to a change of kinetic energy of eddies and by the radial vortices detached from vortex rim vanes.

Centrifugal vortex pumps generate an increased head for a maximum of $23.13 \%$ (average increase of $11.64 \%$ ) compared to the centrifugal pumps with the same geometry at the same angular velocity. Furthermore, the vortex rim of the centrifugal vortex pump increases the amount of the head for working range from 0 to $0.57 Q_{\max }$, and improves the character of $Q$ $H$ characteristics and the stability of the pump. At higher flow rates, it does not affect the level of head. In the same working range the efficiency of the centrifugal vortex is increased, while at the higher flow rates, efficiency is actually lower than of the similar centrifugal pump. It can be concluded that at higher flow rates centrifugal vortex pump wastes driving energy on the spinning vortex rim. At lower flow rates, the contribution of vortex rotor to the head is significant while it does not consume additional driving energy. This suggested that a part of the energy that otherwise is lost in centrifugal pumps is recovered by coherent structures.

\section{Nomenclature}

\begin{tabular}{|c|c|c|c|}
\hline$H$ & Delivery head & $F$ & Weight functions \\
\hline$\Delta p$ & Pressure loss & $Q$ & Flow rate \\
\hline$k_{L}$ & Loss coefficient & $\Delta V_{i}$ & Volume of $i^{\text {th }}$ cell \\
\hline$\rho$ & Density & $N$ & Number of cells \\
\hline$v$ & Velocity & $p$ & Apparent order of accuracy \\
\hline$C_{d}$ & Drag coefficient & $\Phi_{i}$ & Result on the $\mathrm{i}^{\text {th }}$ mesh \\
\hline$\tilde{d}_{w}$ & DES limiter & $r_{i j}$ & $\begin{array}{l}\text { Ratio between result on the } i^{\text {th }} \text { and } j^{\text {th }} \\
\text { mesh }\end{array}$ \\
\hline$d_{\mathrm{w}}$ & Distance to the nearest wall & $\varepsilon_{i j}$ & $\begin{array}{l}\text { Difference between result on the } i^{\text {th }} \\
\text { and } j^{\text {th }} \text { mesh }\end{array}$ \\
\hline$\Delta_{\max }$ & Biggest length of the cell & $e r_{a}$ & Relative estimated error \\
\hline$C_{\text {des }}$ & DES constant & $e r_{\text {ext }}$ & Relative extrapolated error \\
\hline$\varepsilon$ & Dissipation of turbulent kinetic energy & $\mathrm{GCl}$ & Grid convergence index \\
\hline$\beta^{*}$ & SST $k-\omega$ constant & $\operatorname{Re}$ & Reynolds number \\
\hline$k$ & Turbulent kinetic energy & $\tau_{i j}^{\mathrm{R}}$ & Reynolds stress tensor \\
\hline$\omega$ & Rate of energy dissipation & $L_{t}$ & Length of mixing \\
\hline
\end{tabular}

\section{References}

[1] Mihajlovič P.O., Jurevič M.I., Borisovič K.P., Isaakovič R.A., Pavlovič T.I. (2001): “New rotary-vortex pumps for crude oil production in the complicated conditions", in 1. 
International conference of technical sciences, 17-22 September 2001., Rusija, Voronež, pp. 25-37.

[2] Isaakovič R.A, Vasiljevič G.N. (2003): “High-head, economical modifications of multistage centrifugal pump for oil production", in 2. International conference of technical sciences, 15-20 September 2003, Rusija, Voronež, pp. 125-140.

[3] Karakulov S.T., Meljnikov D.J., Pereljmman M.O., Dengaev A.V. (2005): “Ways of increasing efficiency of oil exploitation from oil wells", in 3. International conference of technical sciences, Rusija, Voronež, 14-17 September 2005, pp. 78-87.

[4] Melzi E. (2008), "Vortex impeller for centrifugal fluid-dynamic pumps", European Patent Application EP1 961965 A2, 2008, Italy.

[5] Bilus I., Predin A. (2009): "Numerical and experimental approach to cavitation surge obstruction in water pump", International Journal of Numerical Methods for Heat \& Fluid Flow, Vol. 19 Iss: 7, pp.818 - 834.

[6] Kaupert K.A., Staubli T. (2003), "The Unsteady Pressure Flow in a High Specific Speed Centrifugal Pump Impeller - Part II: Large Eddy Simulations", J. Fluids Eng. 125, Vol. 73

[7] Tucker P.G., (1997) "Numerical precision and dissipation errors in rotating flows", International Journal of Numerical Methods for Heat \& Fluid Flow, Vol. 7 Iss: 7, pp.647 $-658$.

[8] Fluent Inc (2008), "Fluent 12 user guide".

[9] Benim A.C., Escudier M.P., Nahavandi A., Nickson A.K., Syed K.J., Joos F., (2010) "Experimental and numerical investigation of isothermal flow in an idealized swirl combustor", International Journal of Numerical Methods for Heat \& Fluid Flow, Vol. 20 Iss: 3 , pp.348 - 370.

[10] Spalart P.R. (2009), "Detached-eddy simulation", Annual Review of Fluid Mechanics, Vol. 41(1), pp.181-202.

[11] Travin A., Shur M., Strelets M., Spalart P.R. (1999), “Detached eddy simulations past a circular cylinder", Flow, Turbulence and Combustion, Vol. 63, pp.293-313.

[12] Fernández Oro J.M., Argüelles Diaz K.M., Santolaria Morros C., Galdo Vega M., (2011) "Numerical simulation of the unsteady stator-rotor interaction in a low-speed axial fan including experimental validation", International Journal of Numerical Methods for Heat \& Fluid Flow, Vol. 21 Iss: 2, pp.168 - 197.

[13] Shur M.I., Spalart P.R., Strelets M., Travin A. (1999), "Dettached-eddy simulation of an airfoil at high angle of attack", Engineering Turbulence Modelling and Experiments 4, pp. 669-678.

[14] Travin A., Shur M., Strelets M., Spalart P.R. (2002), "Physical and Numerical Upgrades in the Detached-Eddy Simulation of Complex Turbulent Flows", Advances in LES of Complex Flows, Kluwer Academic Publishers, pp. 239-254.

[15] Menter F.R., Kuntz M. (2004), "Adaptation of eddy-viscosity turbulence models to unsteady separated flow behind vehicles", The Aerodynamics of Heavy Vehicles: Trucks, Buses, and Trains, Springer, pp. 339-352.

[16] Launder B.E., Spalding D.B. (1972), "Lectures in Mathematical Models of Turbulence", Academic Press, London.

[17] Feng J., Benra F.K. and Dohmen H.J. (2009), "Comparison of Periodic Flow Fileds in a Radial Pump among CFD, PIV and LDV Results", International Journal of Rotating Machinery, Volume 2009.

[18] Roache P.J.(1997), "Quantification of uncertainty in computational fluid dynamics", Annual Rev. Fluid Mech, pp. 123-160. 
[19] Nuclear energy agency - committee on the safety of nuclear installations (2007), "Best Practice Guidelines for the use of CFD in Nuclear Reactor Safety Applications", $N E A / C S N I / R(2007)$, France.

[20] Dochterman R.W., General Electric Company (1974), "Centrifugal-vortex pump", United States Patent 3,936,240, USA.

[21] Lobanoff V.S., Ross R.R. (1992), "Centrifugal pumps - Design \& Application, 2nd edition", Butterworth-Heinemann, USA.

[22] Mihalić T., Guzović Z., Sviderek S. (2011), “Improving centrifugal pump by adding vortex rotor", Journal of Energy Technology, Slovenia, pp. 11-20.

[23] Gülich J. F. (2008), "Centrifugal Pumps", Springer Berlin Heidelberg New York.

[20] Matijašević B., Sviderek S., Mihalić T. (2006), "Numerical Investigation of the Flow instabilities in Centrifugal Fan", WSEAS Conference on Fluid Mechanics and Aerodynamics, Agios Nicolaos, Greece, pp. 87-98. 